\title{
Roughness of Microspheres for Force Measurements
}

\author{
P. J. van Zwol, ${ }^{\dagger}$ G. Palasantzas, ${ }^{*}{ }^{\dagger}$ M. van de Schootbrugge,${ }^{\dagger}$ J. Th. M. de Hosson, ${ }^{\dagger}$ and \\ V. S. J. Craig*,*
}

Department of Applied Physics, Materials Innovation Institute and Zernike Institute for Advanced Materials, University of Groningen, Nijenborgh 4, 9747 AG Groningen, The Netherlands, and Department of Applied Mathematics, Australian National University, Canberra 0200 ACT, Australia

Received March 3, 2008. Revised Manuscript Received April 21, 2008

\begin{abstract}
We have investigated the morphology and surface roughness of several commercially available microspheres to determine their suitability for force measurements using the atomic force microscope. The roughness varies considerably, depending on sphere size and material, ranging from nearly ideally flat up to micrometer-sized features. Because surface roughness significantly influences the magnitude and accuracy of measurement of surface forces, the results presented here should be helpful for colloid physicists and in particular for those performing force measurements.
\end{abstract}

\section{Introduction}

With the advent of the atomic force microscope (AFM) as a force measurement device ${ }^{1}$ and therefore an easy-to-use alternative to the surface force apparatus, ${ }^{2}$ microspheres have been used for more than a decade to control the geometry and chemistry of the interaction. ${ }^{3,4}$ Because the forces are often very shortranged, it is important that the surfaces are smooth; for example, measurements of dispersion (van der Waals and Casimir) and DLVO forces in the sub-100-nm regime require the use of extremely smooth surfaces. $^{5,6}$ Often size-calibrated, NISTtraceable spheres are used for force measurements (Figure 1), where sphere diameter and local curvature can be measured with a scanning electron microscope (SEM) or an AFM. ${ }^{7-10}$ It is known that the local curvature of microspheres can deviate from the global value reported by the manufacturer. Nevertheless, the local curvature of the portion of the sphere that is involved in the force measurement can be measured using the reverse imaging technique that we have also employed here (Figure 2). ${ }^{10}$

Local curvature deviations together with the surface roughness of the sphere limit the range and precision of force measurements performed with these probes. Besides the fact that surface roughness can significantly alter the magnitude of surface forces, ${ }^{8}$ surface roughness leads to uncertainty in the distance scale as the absolute separation is usually determined from the compliance region that corresponds to the contact point subtracting any deformation of the surface. This limits the accuracy of the force measurement because it is impossible to know how this contact relates to the position of the interface on a rough surface unless the precise features of the surface in the contact region are known. Additionally, describing a rough interface that has a true $z$ profile with a plane that describes an infinitely sharp boundary is problematic.

* Corresponding authors. E-mail: g.palasantzas@rug.nl, vince.craig@anu.edu.au.

+University of Groningen.

* Australian National University.

(1) Burnham, N. A.; Dominguez, D. D.; Mowery, R. L.; Colton, R. J. Phys. Rev. Lett. 1990, 64, 1931.

(2) Israelachvili, J.; Tabor, D. Proc. R. Soc. Lond. A 1972, 331, 19.

(3) Ducker, W. A.; Senden, T. J.; Pashley, R. M. Nature 1991, 353, 239.

(4) Butt, H.-J. Biophys. J. 1991, 60, 1438.

(5) Ederth, T. Phys. Rev. A 2000, 62, 062104.

(6) Katan,A. J. PhD Thesis, ch. 7, pg. 83, https://openaccess.leidenuniv.nl.

(7) Harris, B. W.; Chen, F.; Mohideen, U. Phys. Rev. A. 2000, 62, 052109.

(8) van Zwol, P. J.; Palasantzas, G.; De Hosson, J. T. M. Phys. Rev. B. 2008, 77,075412 .

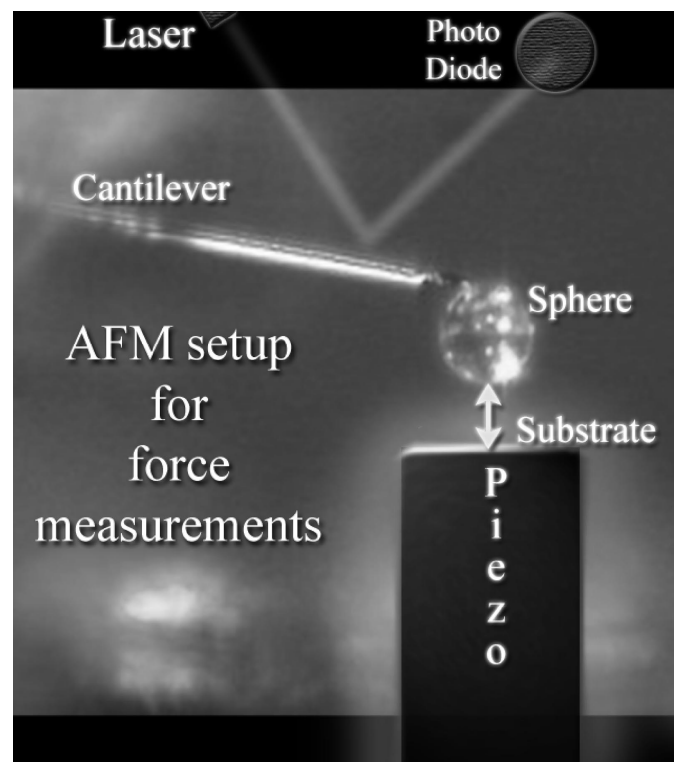

Figure 1. Schematic of the AFM-based force measurement.

In Figure 3, we compare the Casimir force for a rough surface and a smooth surface. Measurements on the rough surface are limited to separations above $50 \mathrm{~nm}$ as a result of the height of the surface peaks. Additionally, the magnitude of the Casimir force is altered by roughness at smaller separations. Measuring the Casimir force at small separations with high precision attracts interest in the search for new forces. ${ }^{11}$ In addition, finite conductivity effects for the Casimir force will be larger at smaller separations, further underlining the importance of the present effort. $^{12,13}$

Furthermore, another related example of the influence of surface roughness is the hydrodynamic force opposing the movement of a sphere near a wall during operation in liquid environments. ${ }^{14-16}$ In Figure 4, the measured hydrodynamic repulsive force for a

(9) van Zwol, P. J.; Palasantzas, G.; De Hosson, J. T. M. Appl. Phys. Lett. 2008, 92, 054101.

(10) Neto, C.; Craig, V. S. J. Langmuir 2001, 17, 2097.

(11) Onofrio, R. New J. Phys. 2006, 8, 237.

(12) Iannuzzi, D.; Lisanti, M.; Capasso, F. PNAS. 2005, 102, 11989.

(13) Svetovoy, V. B.; van Zwol, P. J.; Palasantzas, G.; De Hosson, J. T. M. Phys. Rev. B. 2008, 77, 035439.

(14) Kunert, C. Phys. Rev. Lett. 2007, 99, 176001. 


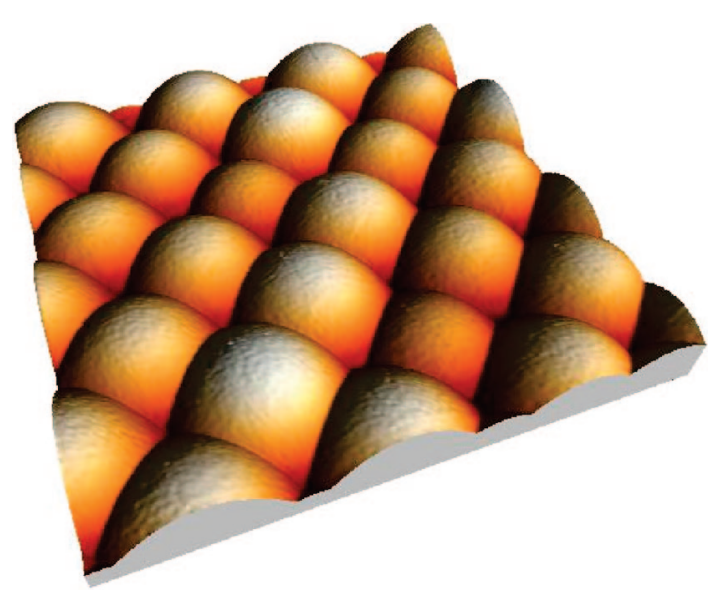

Figure 2. AFM image of a single sphere obtained using the reverse imaging technique ${ }^{10}\left(10 \times 10 \mu \mathrm{m}^{2}\right.$ scan $)$. The sphere is attached to a cantilever and scans a plate with sharp tips in an array (TGT01 NTMDT). Each bump in the image is an independent image of the same part of a single sphere made against a different tip in the array. The portion of the sphere that participates in the force measurement is therefore the part that is imaged.

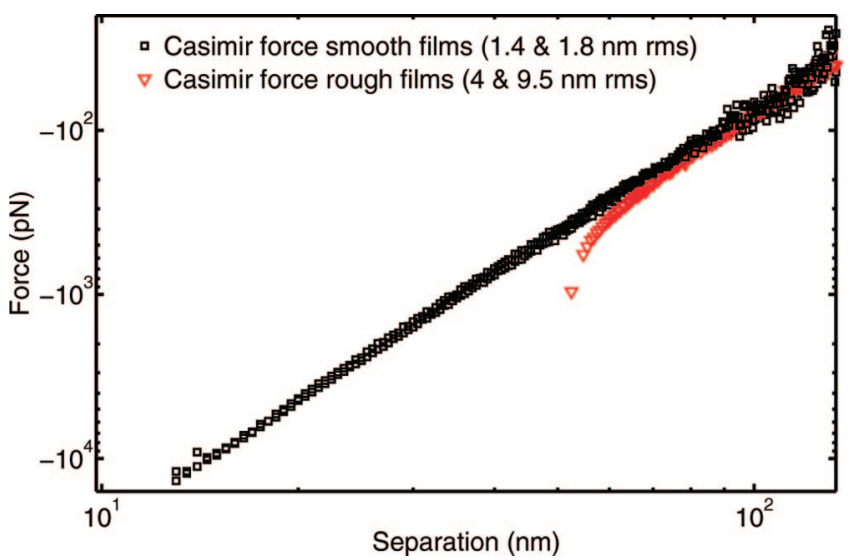

Figure 3. Casimir force measurement under ambient conditions between a $100 \mu \mathrm{m}$ gold-coated polysterene sphere and a Si plate, for rough and smooth surfaces. The point of contact for the smooth surface is $7 \mathrm{~nm}$ (not shown here), and for the rough surface it is $51 \mathrm{~nm}$. See ref 8 .

sphere approaching a surface in a viscous Newtonian solution is shown for surfaces of different roughnesses. Two substrates are compared; the smoother surface $(0.7 \mathrm{~nm} \mathrm{rms}$ roughness $)$ results in a higher hydrodynamic force when compared to a rougher surface (12 $\mathrm{nm}$ rms roughness). ${ }^{17}$

Note that the presence of surface roughness greatly complicates the theoretical analysis of such data as the definition of surface separation becomes ambiguous. In some experiments, the point of zero separation has been defined by the contact of the surfaces, whereas this is the most likely contact over some asperities. Unfortunately, manufacturers of microspheres rarely report the surface roughness, and a consistent study of the roughness of several types of spheres is still missing. This will be the topic of the present article.

\section{Experimental Procedure}

Here we investigate the roughness and global morphology of size-calibrated (NIST-traceable) and noncalibrated, commercially available microspheres by employing AFM (Dimension 3100, Nanoscope Multimode III) and SEM. For the smaller spheres (radius $<40 \mu \mathrm{m}$ ), the reverse imaging technique ${ }^{10}$ will be used. For the larger spheres (radius $>50 \mu \mathrm{m}$ ), we will use normal tapping or

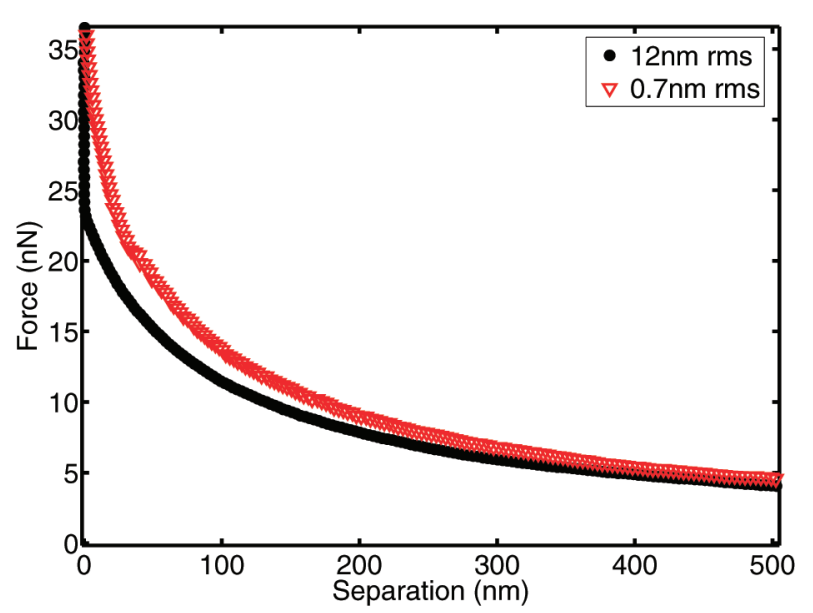

Figure 4. Hydrodynamic repulsive force measured using an atomic force microscope as a borosilicate sphere (rms roughness $0.8 \mathrm{~nm}$ ) of radius $12.55 \mu \mathrm{m}$ is driven toward a silica surface of $0.7 \mathrm{~nm} \mathrm{rms} \mathrm{roughness}$ (upper curve) or a silica surface of $12.0 \mathrm{~nm}$ rms roughness (lower curve) in an aqueous sucrose solution of viscosity $19.2 \mathrm{mPas}$. The drive rate in each case was the same at $43.2 \mu \mathrm{m} \mathrm{s}^{-1}$. The approach rate of the surfaces decreases with decreasing separation as the cantilever spring deflects under the action of the repulsive hydrodynamic force.

Table 1. List of Details for the Spheres Investigated in This Study

\begin{tabular}{|c|c|c|c|}
\hline sphere type & solution & $D_{\mathrm{sph}}(\mu \mathrm{m})$ & $\begin{array}{l}\text { roughness } \\
(\mathrm{nm})\end{array}$ \\
\hline \multicolumn{4}{|c|}{ Large Spheres $\left(4 \times 4 \mu \mathrm{m}^{2}\right.$ Scans $)$} \\
\hline 1 Duke polysterene $4310 \mathrm{~A}$ & liquid & $100 \pm 1.5$ & $1 \pm 0.2(0.5)$ \\
\hline 2 Duke polysterene DVB 4320A & dry & $200 \pm 4$ & $9 \pm 2.5$ \\
\hline 3 Duke polysterene DVB 4330A & dry & $300 \pm 6$ & $40 \pm 10$ \\
\hline 4 Duke sodalime glass 9100 & dry & $100 \pm 5$ & $30 \pm 15$ \\
\hline 5 Kisker sodalime glass PGB 15 & dry & $50-150$ & $25 \pm 15$ \\
\hline 6 Kisker PMMA PLPM 100 & dry & $100-200$ & $2.8 \pm 0.7$ \\
\hline \multicolumn{4}{|c|}{ Small Spheres $\left(1 \times 1 \mu \mathrm{m}^{2}\right.$ Scans $)$} \\
\hline 7 Duke sodalime glass 9030 & dry & $30 \pm 2.1$ & $5.5 \pm 1.5$ \\
\hline 8 Duke borosilicate glass 9020 & dry & $20 \pm 1.4$ & $0.7 \pm 0.1$ \\
\hline 9 BioForce tungsten & dry & $\sim 10$ & very rough \\
\hline 10 Polysciences Fe, cat no. 19829 & dry & $\sim 10$ & very rough \\
\hline 11 Corpuscular $\mathrm{Al}_{2} \mathrm{O}_{3}$, C-ALu-20.0 & 0 liquid & 20 & $12 \pm 1$ \\
\hline 12 Corpuscular $\mathrm{TiO}_{2}$, C-Tio-22.0 & liquid & 22 & 4.5 \\
\hline
\end{tabular}

contact mode AFM. For all spheres, SEM pictures are provided. For standard SEM imaging, the spheres are coated with a thin metallic layer to reduce charging. This does not change the global morphology; however, it increases the surface roughness. Therefore, AFM scans of the spheres were carried out prior to the SEM studies or on separate samples.

Note that the larger spheres are useful for force measurements in air or vacuum where higher force sensitivity may be required, whereas the smaller spheres are more useful for dynamic mode AFM or for force measurements in liquids. Table 1 shows the rms roughness of the spheres together with the standard deviation found for all of the AFM scans. The numbers in Table 1 correspond to the numbers in Figures 5 and 6 containing the AFM and SEM images.

\section{Results of Morphology Analysis}

Large Spheres. For each type of sphere, 10 or more AFM $\left(4 \times 4 \mu \mathrm{m}^{2}\right)$ scans and some SEM pictures were taken. Sphere 1 has a very well defined NIST-traceable diameter (within 1.5\%). It is also very smooth with $1 \mathrm{~nm}$ roughness rms over a $4 \times 4$ $\mu \mathrm{m}^{2}$ area, whereas locally $\left(1 \times 1 \mu \mathrm{m}^{2}\right)$ it is smoother down to $0.5 \mathrm{~nm}$ rms and it has no evident surface features. The sphere is, in fact, so smooth that contributions from inaccuracies related to the AFM piezo (which is not a closed loop) or the flattening procedure may dominate the measured roughness. A disadvantage 

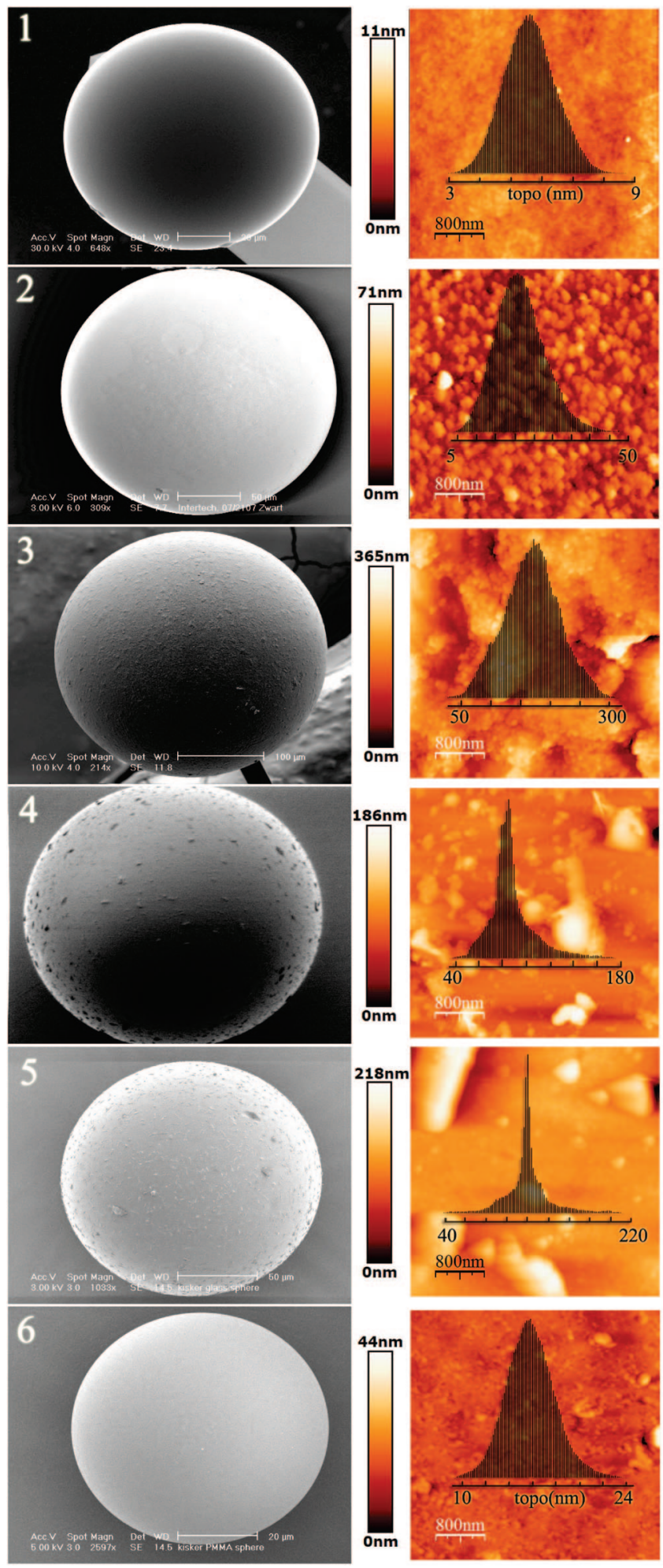

Figure 5. SEM pictures and AFM scans (with height topography) are shown for six larger (radius $>50 \mu \mathrm{m}$ ) spheres. Global details can be seen from SEM, and local features can be determined from the AFM scans. Sphere details are given in Table 1.

of these extremely smooth spheres is that they are supplied as a dispersion in an aqueous solution, making it hard to attach them in a clean manner to a cantilever. An effective approach in dealing with spheres in solutions is to disperse a small volume of the solution containing a number of spheres in a volatile purified solvent such as ethanol. The ethanol quickly evaporates, leaving behind the dry spheres.
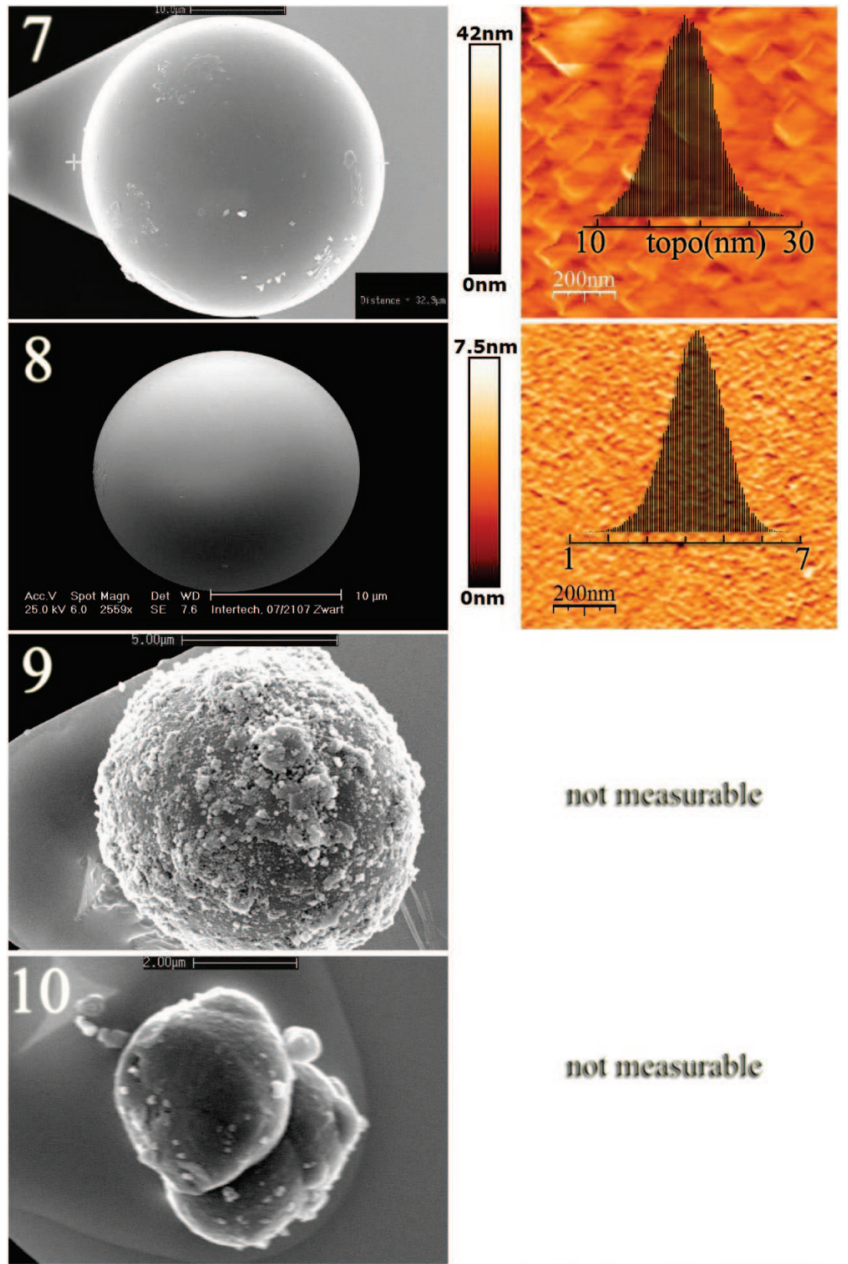

not measurable
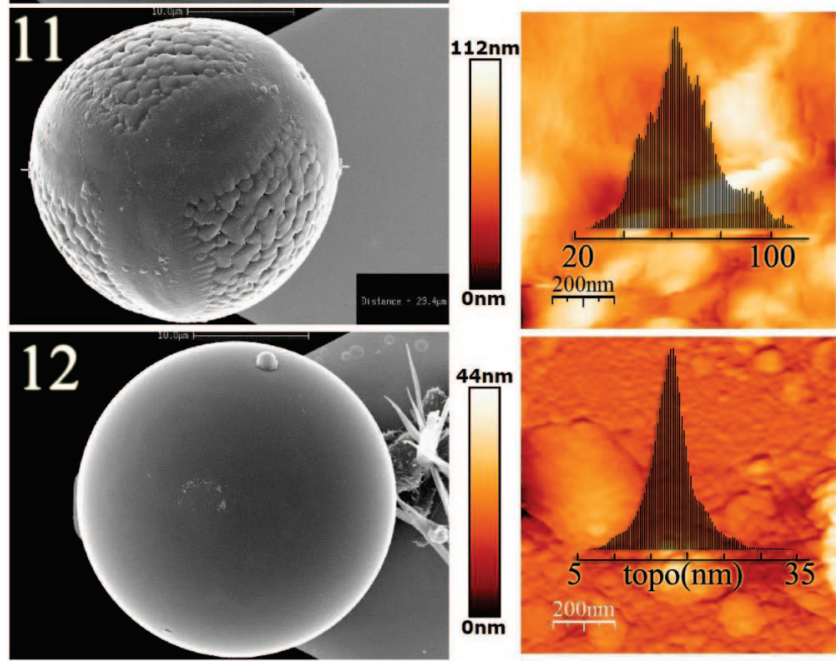

Figure 6. SEM pictures and AFM scans (with height topography) shown for six smaller (radius $<40 \mu \mathrm{m}$ ) spheres. Global details can be seen from SEM, and local features can be determined from the AFM scans. Sphere details are given in Table 1.

We found that the larger polysterene DVB spheres (spheres 2 and 3 in Figure 5) have a much higher roughness and are therefore less useful in close-range measurements. Note, however, that using smooth spheres on soft cantilevers may lead to stiction problems. ${ }^{18}$ Therefore, sphere 2 would still suffice for measurements at separations above $50 \mathrm{~nm}$. Duke sodalime glass spheres

(15) McHale, G.; Newton, M. I. J. Appl. Phys. 2004, 95, 373. 
(sphere 4 in Figure 5) are too rough and even have micrometersized features. The glass and PMMA spheres from KISKER (spheres 5 and 6, respectively, in Figure 5) were not NIST calibrated. The KISKER sodalime glass spheres have a surface roughness similar to those of materials supplied by Duke. The PMMA spheres with $2.8 \mathrm{~nm} \mathrm{rms}$ roughness on average are quite smooth and clean over large areas, but they have some local (nanosized) features. The PMMA spheres are supplied dry, which makes them a good replacement for sphere 1 if data obtained at extremely close proximity (separations $<20 \mathrm{~nm}$ ) are not necessary. We have also measured PMMA (Kisker spheres PMMA plpm $400,600,700)$ with diameters ranging from $300 \mu \mathrm{m}$ to $2 \mathrm{~mm}$. For all of these spheres, we found that in regions without local features the roughness varied between 3 and $5 \mathrm{~nm}$ rms. However, for spheres larger than $500 \mu \mathrm{m}$, local features tended to be more frequent, wider in area, and as large as $200 \mathrm{~nm}$. Nonetheless the PMMA plpm 400 spheres can still be useful for force measurements because they were relatively clean.

Small Spheres. Small spheres are difficult to image using conventional AFM imaging. Therefore, for the small spheres investigated here we used the reverse imaging technique ${ }^{10}$ (Figure 2). The scan size used to determine the rms roughness was reduced to $1 \mu \mathrm{m}$ because the curvature of the spheres ensures that images over larger scan sizes are difficult to obtain without artifacts. The smaller sodalime glass spheres (sphere 7, Figure 6) have lower roughness than the larger spheres described above (spheres 5 and 6), but the roughness is still significant. These spheres also have easily identifiable local features. In sharp contrast to the sodalime glass spheres, the borosilicate glass spheres (sphere 8, Figure 6) are extremely smooth with a roughness of $0.7 \mathrm{~nm} \mathrm{rms}$ and a peak-to-peak roughness of $4 \mathrm{~nm}$ top to bottom; these are the smoothest spheres that we have found.

The tungsten and iron spheres (spheres 9 and 10, Figure 6) are extremely rough and far from spherical. Such roughness precludes obtaining meaningful AFM images using the reverse imaging technique. The alumina spheres (sphere 11, Figure 6) show clear evidence of a rich internal structure and significant deviations from sphericity, although on the smoother portion of the surface the roughness is only about $12 \mathrm{~nm}$ rms. Regardless, these spheres are not suitable for surface force measurements. We found, however, that the titania spheres (sphere 12, Figure 6) are reasonably good quality; however, with $4.5 \mathrm{~nm} \mathrm{rms}$ roughness, measurements at short range $(<30 \mathrm{~nm})$ would need to be treated with caution. In summary, the borosilicate spheres are ideal for force measurements, and the Titania spheres are also possible to use if this particular surface chemistry is required.

Finally, to confirm whether the surface roughness is consistent from batch to batch we bought a second batch of the $20 \mu \mathrm{m}$ borosilicate spheres and found no difference between the batches. We also found that the sodalime glass microspheres from Kisker have the same features as those from Duke. Note also that the roughness of the $200 \mu \mathrm{m}$ polysterene spheres found here is the same as that reported previously. ${ }^{12}$ Furthermore, small polysterene spheres with a diameter $<50 \mu \mathrm{m}$ as measured here ${ }^{19}$ also have an rms roughness of $1 \mathrm{~nm}$, which is consistent with our findings. Although these last examples from independent studies do not provide strong statistical evidence, they do indicate that the surface roughness of spheres of the same material is

(16) Bonaccurso, E.; Butt, H. J.; Craig, V. S. J. Phys. Rev. Lett. 2003, 90, 144501 . consistent and reproducible from batch to batch. Nevertheless, the data provided here can be used as a guideline for others performing force measurements at separations of $<1 \mu \mathrm{m}$.

\section{Discussion}

Having measured the roughness of the sphere and that of the surface for an experimental system, we will discuss briefly how these factors relate to the separation distance upon contact (Figure 1). Indeed, the latter corresponds to the peak distribution of the roughness and varies consistently with it. For example, for Casimir force measurements between gold surfaces, ${ }^{8,9}$ it has been found to vary almost linearly with the sum of the rms roughness amplitudes of sphere and substrate with a proportionality factor having a value of $\sim 3$ to 4 . Therefore, caution is required because the actual possible separation is significantly larger than the rms roughness amplitude. Moreover, the determination of the separation upon contact with accuracy better than $1 \mathrm{~nm}$ is necessary if high accuracy measurements of the Casimir force at close separations $(<20 \mathrm{~nm})$ are necessary. 8,9

As a final remark, we note that in some cases metalized spheres are needed. However, the roughness of the sphere increases significantly when it is metalized by evaporation or sputtering. In addition, some (polymer) spheres cannot withstand aggressive plasma treatment. Therefore, a viable option may be the use of atomic layer deposition (ALD) ${ }^{20}$ which is known to give smooth metal (and metal oxide) surfaces on Si wafers (rms $<1 \mathrm{~nm}$ ). Likewise, the smooth Bo-Si spheres investigated here may also be employed as substrates for coating by ALD.

\section{Conclusions}

The roughness of microspheres, which is often used in force measurements, varies greatly with type. Some spheres are as smooth as silicon wafers, and others are comparatively rough. Polysterene and PMMA spheres are quite smooth, whereas sodalime glass and small alumina and metallic spheres are rough. The borosilicate spheres are the smoothest spheres that we have investigated. They are supplied dry and can withstand high temperature, which makes them very suitable for several coatings used in industrial applications. Because roughness limits the measurement range and the precision to which the absolute separation is known, it is one of the most important parameters in choosing a sphere for making colloid probes. Some smooth spheres found here are very smooth and as such are very useful for force measurements in general. In any case, the data provided here can be used as a guideline for others performing force measurements at separations $<1 \mu \mathrm{m}$.

Acknowledgment. The research was carried out under project number MC3.05242 in the framework of the Strategic Research Programme of the Materials Innovation Institute (M2I) at the former Netherlands Institute for Metals Research (NIMR). P.J.v.Z., G.P., M.v.d.S., and J.Th.M.d.H. gratefully acknowledge financial support from the M2I. V.S.J.C. gratefully acknowledges the support of the Australian Research Council.

\section{LA800664F}

(17) Munday, J.; Capasso, F. Phys Rev. A. 2007, 75, 060102.

(18) van Zwol, P. J.; Palasantzas, G.; De Hosson, J. T. M. Appl. Phys. Lett. 2007, 91, 101905.

(19) Jourdan, G.; Lambrecht, A.; Comin, F.; Chevrier, J. arXiv: 2007, 0712 , 1767 The authors state that the roughness of the sphere after coating $300 \mathrm{~nm}$ gold is $<3 \mathrm{~nm} \mathrm{rms}$. In a private communication the authors have confirmed that the rms roughness off their polysterene spheres was $1 \mathrm{~nm}$ rms prior to coating.

(20) Lim, S.; Rahtu, A.; Gordon, R. G. Nat. Mater. 2003, 2, 749. 